\title{
GENERATION AND STABILITY OF SUBHARMONIC AND MODULATED SUBHARMONIC OSCILLATIONS IN NONLINEAR SYSTEMS*
}

\author{
BY \\ B. J. MATKOWSKY, E. H. ROGERS AND L. A. RUBENFELD) \\ Rensselaer Polytechnic Institute
}

Introduction. Electrical, mechanical, and other systems whose bohavior is gorerned by linear equations with constant coefficients have the property that the only possible forced periodic oscillations are those whose frequency is exactly that of the forcing function. In sharp contrast, systems governed by nonlinear equations exhibit a wide variety of periodic oscillations in addition to those having the same period as the driving function. Among these is the so-called subharmonic resonance in which the driving frequency is an integer multiple of the response frequency. In radio circuits this plienomenon is also referred to as frequency demultiplication. Such phenomena have been observed in numerous experiments (see, e.g., the references in [8]).

The problem of subharmonic resonance has been investigated, among others, by von Karman [1], whose interest in the problem was due to the fact that certain parts of an airplane can be excited to violent oscillations by an engine ruming witis a number of revolutions much larger than the natural frequencies of the oscillating parts. In this connection Lefshetz [2] describes a commercial plane in which the propellors inciuced a double-period vibration in the wings which in turn induced a quadruple-period vibration in the rudder. The last vibration was so violent that the plane broke up. There have be'n a number of mathematical investigations of subharmonic resonances. For a partial list, of these works one may consult the references in [3]-[6]. We mention specifically, however, the works of Cohen ([7] and [8]) which are most closely related to ours. He considered the steady-state problem of finding the possible subharmonic resonances.

In this paper we consider the initial-value problem for the sliglitly nonlinear forced van der Pol equation in order to see how the subharmonics are generated from arbitrary initial data and also to determine their stability. Our discussion is clearly applicable to a large class of nonlinear problems for which the present analysis serves as a prototype. We find that the solution is characterized by two time scales. One is a fast time, which characterizes the period of the resulting oscillations, while the other, a slow tims, describes the evolution of the initial data into that oscillation.

An interesting new phenomenon which we find is the possible occurrence of modulated subharmonic oscillations. These are oscillations whose frequency differs slightly from that of the subharmonic. Note that frequency perturbation is equivalent to a periodic amplitude modulation. In the case of modulated subharmonics, the slow time characterizes not only the time of evolution of the solution to its final state, but also the period

* Received September 19, 1971. The research reported in this paper was supported in part by the National Science Foundation Grants GP-28247 and GP-27368. 
of the modulating amplitude. It is instructive to visualize these oscillations by considering the phase plane for the amplitude function. In the case of subharmonics, every point on a certain circle in the phase plane is an equilibrium point and the circle of equilibrium points is stable. In the case of modulated subharmonics, the circle becomes a limit cycle and this limit cycle is stable. Employing the Poincaré-Bendixson theorem, Stoker [3] has proved the existence of such a limit cycle for the problem of harmonic resonance, though not for subharmonic resonance. In this paper we are able to calculate the limit cycle, or more generally the eventual steady-state behavior of the system, the approach to the steady state and its stability.

The modulated subharmonics which we find are related to the notion of subharmonic synchronization in which the response frequency locks in or is entrained at a definite frequency, though the forcing frequency may vary in some range. Thus modulated subharmonics will be obtained for a band of frequencies about the natural frequency of the linearized equation. It is worth mentioning that subharmonic synchronization has been found useful for very accurate speed control of electric motors such as those used in high-precision clocks and similar devices in which an extreme accuracy of speed control is necessary.

Formulation. We consider the initial-value problem for the forced van der Pol equation

$$
\begin{gathered}
\ddot{x}-\epsilon \dot{x}\left(1-x^{2}\right)+\omega_{0}^{2} x=F \cos \left(n \omega t^{\prime}+\phi\right), \\
x(0)=A, \quad \dot{x}(0)=B,
\end{gathered}
$$

where "." denotes $d / d t^{\prime}, \epsilon$ is a small positive parameter, $F$ and $\phi$ are the constant amplitude and phase respectively of the forcing term, and $n$ is a positive integer other than one or three. The reason for excluding the case $n=3$ from these considerations will be discussed below. The constant $\omega$ in the forcing term is assumed to be close to $\omega_{0}$, the natural frequency of the system when $\epsilon=0$. We seek a solution of (1) for small values of $\epsilon$. For large time, we expect to recover from our solution the steady-state results previously obtained. In addition to retrieving known steady-state results, we also obtain steady-state solutions which are apparently new. These solutions are the modulated subharmonics referred to above.

We treat (1), using the method of multiple time scales [9]. Thus we seek solutions of the form

$$
x(t ; \epsilon) \sim \sum_{i=0}^{\infty} u^{i}(t, \tau) \epsilon^{j},
$$

where

$$
\begin{aligned}
t & =\omega t^{\prime}, \\
\tau & =\epsilon t^{\prime},
\end{aligned}
$$

with

$$
\omega \sim \sum_{i=0}^{\infty} \omega_{i} \epsilon^{i} .
$$

In (2) and (5) the symbol $\sim$ denotes asymptotic equivalence. Then, inserting (2) into (1) and setting the coefficient of each power of $\epsilon$ separately to zero, we obtain the following 
system of initial-value problems to determine the coefficients $u^{i}(t, \tau)$ and the numbers $\omega_{i}$ :

$$
\begin{gathered}
L u^{0} \equiv \omega_{0}^{2}\left(u_{t t}^{0}+u^{0}\right)=F \cos (n t+\phi), \\
u^{0}(0,0)=A, \quad \omega_{0} u_{t}^{0}(0,0)=B, \\
L u^{1}=-2 \omega_{0} \omega_{1} u_{t t}^{0}-2 \omega_{0} u_{t r}^{0}+\omega_{0} u_{t}^{0}\left[1-\left(u^{0}\right)^{2}\right] \equiv r_{1}, \\
u^{1}(0,0)=0, \quad \omega_{0} u_{t}^{1}(0,0)=-u_{\tau}^{0}(0,0)-\omega_{1} u_{t}^{0}(0,0), \\
L u^{k}=r_{k} \equiv-\omega_{0}\left[2 \omega_{1} u_{t t}^{k-1}+2 u_{t \tau}^{k-1}+u_{t}^{k-1}\left\{\left(u^{0}\right)^{2}-1\right\}+2 u^{0} u_{t}^{0} u^{k-1}+2 \omega_{k} u_{t t}^{0}\right]-P_{k} \\
\quad(k=2,3,4, \cdots), \\
u^{k}(0,0)=0, \quad \omega_{0} u_{t}^{k}(0,0)=-Q_{k},
\end{gathered}
$$

where the quantities $P_{k}$ and $Q_{k}$ are defined by

$$
\begin{gathered}
P_{k} \equiv u_{t l}^{0} \sum_{l=1}^{k-1} \omega_{l} \omega_{k-l}+\sum_{m=1}^{k-2} u_{t t}^{m} \sum_{l=0}^{k-m} \omega_{l} \omega_{k-m-l}+2 \sum_{m-1}^{k-1} u_{t \tau}^{m-1} \omega_{k-m} \\
-\sum_{m-1}^{k-1} u_{t}^{m-1} \omega_{k-m}+\left(u^{0}\right)^{2} \sum_{l=1}^{k-1} u_{t}^{l-1} \omega_{k-l} \\
+\omega_{0} u_{l}^{0} \sum_{p=1}^{k-2} u^{p} u^{k-1-p}+\sum_{m=2}^{k-1} \sum_{l=1}^{m} \sum_{p=0}^{k-m} u_{t}^{l-1} u^{p} u^{k-m-p} \omega_{m-l} \\
+u_{\tau}^{k-2}-u_{\tau}^{k-2}+\sum_{m=2}^{k} \sum_{l=0}^{k-m} u_{\tau}^{m-2} u^{l} u^{k-m-l} \\
Q_{k} \equiv u_{\tau}^{k-1}(0,0)+\sum_{m=0}^{k-1} u_{t}^{m}(0,0) \omega_{k-m} .
\end{gathered}
$$

In (9) and (10) $u^{l}$ and $\omega_{l}$ are understood to be zero for $l<0$.

The solution of $(6)$ is

$$
u^{0}(t, \tau)=\alpha_{0}(\tau) e^{i t}+\bar{\alpha}_{0}(\tau) e^{-i t}+\gamma e^{i n t}+\bar{\gamma} e^{i n t},
$$

where "-" denotes complex conjugate, $\boldsymbol{\gamma}$ is given by

$$
\gamma=F e^{i \phi} / 2 \omega_{0}^{2}\left(1-n^{2}\right), \quad(n \neq 1),
$$

and $\alpha_{0}(\tau)$ is a yet-undetermined function of the second variable $\tau$. From (6), the initial condition for $\alpha_{0}(\tau)$ is

$$
\alpha_{0}(0)=\frac{1}{2}\left[(A-2 \operatorname{Re} \gamma)-i\left(\left(B / \omega_{0}\right)+2 n \operatorname{Im} \gamma\right)\right],
$$

where

$$
\operatorname{Re} \gamma=\frac{F \cos \phi}{2 \omega_{0}^{2}\left(1-n^{2}\right)}, \quad \operatorname{Im} \gamma=\frac{F \sin \phi}{2 \omega_{0}^{2}\left(1-n^{2}\right)} .
$$

We note that Eqs. (7) and (8) are inhomogeneous forms of (6). Therefore a necessary condition for bounded solutions to exist is that their right-hand sides satisfy the orthogonality condition

$$
\left(r_{k}, e^{i t}\right)=\left(r_{k}, e^{-i t}\right)=0, \quad k=1,2,3, \cdots,
$$


where

$$
(f, g)=\lim _{T \rightarrow \infty} \frac{1}{T} \int_{0}^{T} f g d t .
$$

This condition is equivalent to the usual condition of casting out secular terms. Condition (15) with $k=1$ implies that $\alpha_{0}(\tau)$ satisfies the following nonlinear ordinary differential equation*:

$$
\alpha_{0}^{\prime}+\left[i \omega_{1}+\frac{1}{2}\left(\left|\alpha_{0}\right|^{2}+2|\gamma|^{2}-1\right)\right] \alpha_{0}=0
$$

with $\alpha_{0}(0)$ given by (13). ( $\left|\alpha_{0}\right|$ and $|\gamma|$ denote the absolute values of these complex quantities.) We mention at this point that if $n=3, \mathrm{Eq}$. (17) must be modified to include additional terms. We do not treat the case $n=3$ here, preferring to defer its consideration to another time.

To find the solution of (17) and (13) we multiply (17) by $\bar{\alpha}_{0}(\tau)$ and add the result to its complex conjugate. This yields the following equation for the quantity $R=\left|\alpha_{0}\right|^{2}$ :

$$
R^{\prime}+R\left(R+2|\gamma|^{2}-1\right)=0 \text {. }
$$

The initial condition for (18) follows from (13). Thus

$$
R \equiv\left|\alpha_{0}\right|^{2}=\left(2|\gamma|^{2}-1\right) /\left(\delta \exp \left\{2|\gamma|^{2}-1\right\} \tau-1\right),
$$

where

$$
\delta=1+\left(\left(2|\gamma|^{2}-1\right) /\left|\alpha_{0}(0)\right|^{2}\right)
$$

Inserting (19) into (17), we find that

$$
\alpha_{0}^{\prime}+\alpha_{0}\left[i \omega_{1}+\frac{1}{2}\left(R+2|\gamma|^{2}-1\right)\right]=0 .
$$

Noting from (18) that

$$
R+2|\gamma|^{2}-1=-(1 / R)(d R / d \tau)
$$

we write (21) as

$$
\left(d \alpha_{0} / \alpha_{0}\right)+i \omega_{1} d \tau-\frac{1}{2}(d R / R)=0
$$

whose solution is

$$
\alpha_{0}(\tau)=k \exp \left(-i \omega_{1} \tau\right)\left[\frac{2|\gamma|^{2}-1}{\delta \exp \left[2|\gamma|^{2}-1\right] \tau-1}\right]^{1 / 2},
$$

where

$$
\begin{aligned}
& \delta=1+\left(\left(2|\gamma|^{2}-1\right) /\left|\alpha_{0}(0)\right|^{2}\right), \\
& k=\alpha_{0}(0) /\left|\alpha_{0}(0)\right|,
\end{aligned}
$$

and $\gamma$ and $\alpha_{0}(0)$ are given by (12) and (13) respectively. It is interesting to do a phase plane analysis of (17). To this end we set

$$
\alpha_{0}=u+i v \text {. }
$$

* The two orthogonality conditions (15) actually yield a second equation which can be obtained from (17) by taking complex conjugates. 
Then, from (17), $u$ and $v$ satisfy

$$
\begin{aligned}
& u^{\prime}=-\frac{1}{2} u\left(u^{2}+v^{2}+2|\gamma|^{2}-1\right)+\omega_{1} v, \\
& v^{\prime}=-\frac{1}{2} v\left(u^{2}+v^{2}+2|\gamma|^{2}-1\right)-\omega_{1} u .
\end{aligned}
$$

We note that for $\omega_{1} \neq 0$ the only singular point of (28) is the origin, which is a center for the linearized problem. From the solution of the nonlinear problem, however, we know that, in the case $2|\gamma|^{2}-1<0$, all solutions move away from the origin and approach a stable limit cycle which can be gotten from (24). The stability of the limit cycle follows immediately from Eq. (18) for $R=\left|\alpha_{0}\right|^{2}=u^{2}+v^{2}$ which states that if $R$ is less (greater) than its steady value $1-2|\gamma|^{2}$, then $R^{\prime}$ is positive (negative), so that $R$ will increase (decrease) to the limit cycle. When $2|\gamma|^{2}-1>0$, all solutions approach the origin. It is of interest to note that a center for the linearized problem does not remain a center for the nonlinear problem.

In the case $\omega_{1}=0$, with $2|\gamma|^{2}-1<0$, all the points on the circle $u^{2}+v^{2}=1-$ $2|\gamma|^{2}$, in addition to the origin, are singular points. Each point on the circle represents a periodic solution. Each has the same period and amplitude and differs only in phase. Arguing as above, we see that the circle of singular points is stable in the sense that if any point on the circle is perturbed, it will return to the circle, though not necessarily to the original point. In this case the origin is an unstable node and the solution proceeds according to (24) to one of the singular points of the circle. For $2|\gamma|^{2}-1>0$, the origin is a stable node and $\alpha_{0}(\tau)$ eventually decays to zero.

Employing (12), (13), and (24) in (11), we find that the leading term $u^{0}(t, \tau)$ in the expansion of $u$ is given by

$$
\begin{aligned}
u^{0}(t, \tau)=\frac{F \cos (n t+\phi)}{\omega_{0}^{2}\left(1-n^{2}\right)}+\frac{1}{\left|\alpha_{0}(0)\right|}\left[\frac{2|\gamma|^{2}-1}{\delta \exp \left[2|\gamma|^{2}-1\right] \tau-1}\right]^{1 / 2} \\
\cdot\left[(A-2 \operatorname{Re} \gamma) \cos \left(t-\omega_{1} \tau\right)+\left(\frac{B}{\omega_{0}}+2 n \operatorname{Im} \gamma\right) \sin \left(t-\omega_{1} \tau\right)\right] .
\end{aligned}
$$

The long time behavior of $u^{0}$ is given by

$$
\begin{gathered}
u^{(0)}(t, \tau)=\frac{F \cos (n t+\phi)}{\omega_{0}^{2}\left(1-n^{2}\right)}+\frac{\left(1-2|\gamma|^{2}\right)^{1 / 2}}{\left|\alpha_{0}(0)\right|} \\
\cdot\left[(A-2 \operatorname{Re} \gamma) \cos \left(t-\omega_{1} \tau\right)+\left(\frac{B}{\omega_{0}}+2 n \operatorname{Im} \gamma\right) \sin \left(t-\omega_{1} \tau\right)\right], \quad|\gamma|^{2}<\frac{1}{2}, \\
u^{0}(t, \tau)=\frac{F \cos (n t+\phi)}{\omega_{0}^{2}\left(1-n^{2}\right)}, \quad|\gamma|^{2}>\frac{1}{2},
\end{gathered}
$$

Thus we see that if $|\gamma|^{2}>\frac{1}{2}$, that is if the forcing amplitude $F$ is "large" compared to $n^{2}$ (cf. (12)), then the steady-state solution for $u^{0}$ is due solely to the forcing term. However, if the forcing amplitude is not too large, then there exists an additional steadystate term. We observe that in the latter case we obtain a subharmonic solution only if $\omega_{1}=0$, whereas $\omega_{1} \neq 0$ corresponds to the above-mentioned modulated subharmonics. We also note that the periodic function given by (30) represents the steady-state behavior of the initial-value problem and this periodic solution is stable. The stability of (30) follows from the fact that a perturbation of (30) leads to a new initial-value problem which differs from the original initial-value problem only in initial data and the phase $\phi$ 
of the forcing function. Therefore, by our previous analysis, the eventual behavior of the new initial-value problem is also given by (30) with only the phase being changed in each of the two terms in (30). In the first term the phase is changed due to the change in $\phi$. In the second term the quantities $(A-2 \operatorname{Re} \gamma)$ and $\left(\left(B / \omega_{0}\right)+2 n \operatorname{Im} \gamma\right)$ are each changed, though the amplitude of this term, given by

$$
\frac{\left(1-2|\gamma|^{2}\right)^{1 / 2}}{\left|\alpha_{0}(0)\right|}\left((A-2 \operatorname{Re} \gamma)^{2}+\left(\frac{B}{\omega_{0}}+2 n \operatorname{Im} \gamma\right)^{2}\right)^{1 / 2}=2\left(1-2|\gamma|^{2}\right)^{1 / 2}
$$

remains unchanged. In Eq. (31) we have made use of (13). The fact that the second term changes only in phase also follows from the phase plane discussion given above. Finally, we observe from (30) and (3)-(5) that to $O\left(\epsilon^{2}\right)$ the frequency of the steady-state solution (for $|\gamma|^{2}<\frac{1}{2}$ ) is independent of $\omega_{1}$, so that for arbitrary $\omega_{1}$ the response frequency is entrained or locked in at $\omega-\epsilon \omega_{1}$.

We shall now determine $\omega_{2}$, which yields the deviation of the response frequency from the natural frequency of the linearized equation. Employing (11) in (7), and solving the resulting differential equation for $u^{1}$, we obtain

$$
u^{1}(t, \tau)=\alpha_{1}(\tau) e^{i t}+\bar{\alpha}_{1}(\tau) e^{-i t}+D(t, \tau)+\bar{D}(t, \tau)
$$

where

$$
\begin{aligned}
D(t, \tau)=\frac{i \alpha_{0}^{8}}{8 \omega_{0}} & e^{3 i t}+\frac{i \alpha_{0} \gamma^{2}(2 n+1)}{\omega_{0}\left[(2 n+1)^{2}-1\right]} e^{i(2 n+1) t}+\frac{i \bar{\alpha}_{0} \gamma^{2}(2 n-1) e^{i(2 n-1) t}}{\omega_{0}\left[(2 n-1)^{2}-1\right]} \\
& +\frac{i \alpha_{0}^{2} \gamma(n+2)}{\omega_{0}\left[(n+2)^{2}-1\right]} e^{i(n+2) t}+\frac{i \bar{\alpha}_{0}^{2}(n-2)}{\omega_{0}\left[(n-2)^{2}-1\right]} e^{i(n-2) t} \\
& +\frac{i n \gamma^{3} e^{3 i n t}}{\omega_{0}\left[9 n^{2}-1\right]}+\frac{n \gamma}{\omega_{0}\left(n^{2}-1\right)}\left[-2 n \omega_{1}+i\left(2\left|\alpha_{0}\right|^{2}+|\gamma|^{2}-1\right)\right] e^{i n t} .
\end{aligned}
$$

The dependence of $D(t, \tau)$ on $\tau$ occurs through $\alpha_{0}(\tau)$. Initial conditions for $\alpha_{1}(\tau)$ can be derived from (7), but we shall not do so since our purpose is to determine $\omega_{2}$. This is done by examining the long-time behavior of $\alpha_{1}(\tau)$. The equation for $\alpha_{1}(\tau)$ is determined by employing the orthogonality condition (15) with $k=2$. We thus find

$$
\alpha_{1}^{\prime}+\alpha_{1}\left[\left|\alpha_{0}\right|^{2}+|\gamma|^{2}-\frac{1}{2}+i \omega_{1}\right]+\frac{1}{2} \alpha_{0}^{2} \bar{\alpha}_{1}=\frac{\alpha_{0}}{2 i \omega_{0}} G+\frac{\gamma^{2} \bar{\alpha}_{0}^{3}}{2 \dot{i} \omega_{0}} \delta_{n 2} c_{1}+\frac{\gamma \bar{\alpha}_{0}^{4} \delta_{n 5}}{2 \dot{\omega} \omega_{0}} c_{2},
$$

where $\delta_{n 2}$ and $\delta_{n s}$ are Kronecker deltas and

$$
\begin{gathered}
G=\left[i \omega_{1}+\frac{1}{2}\left(\left|\alpha_{0}\right|^{2}+2|\gamma|^{2}-1\right)\right]\left[2 i \omega_{1}-1+2\left(\left|\alpha_{0}\right|^{2}+|\gamma|^{2}\right)\right] \\
+\left|\alpha_{0}\right|^{2}\left[-i \omega_{1}+\frac{1}{2}\left(\left|\alpha_{0}\right|^{2}+2|\gamma|^{2}-1\right)\right]-\left[i \omega_{1}+\frac{1}{2}\left(\left|\alpha_{0}\right|^{2}+2|\gamma|^{2}-1\right)\right]^{2} \\
-\frac{1}{2}\left|\alpha_{0}\right|^{2}\left(\left|\alpha_{0}\right|^{2}+2|\gamma|^{2}-1\right)+\omega_{1}^{2}+2 \omega_{0} \omega_{2}-i \omega_{1}\left(\left|\alpha_{0}\right|^{2}+2|\gamma|^{2}-1\right) \\
+\frac{\left|\alpha_{0}\right|^{4}}{8}+\frac{(2 n+1)|\gamma|^{4}}{(2 n+1)^{2}-1}-\frac{(2 n-1)|\gamma|^{4}}{(2 n-1)^{2}-1}+\frac{2(n+2)\left|\alpha_{0}\right|^{2}|\gamma|^{2}}{(n+2)^{2}-1} \\
-\frac{2(n-2)\left|\alpha_{0}\right|^{2}|\gamma|^{2}}{(n-2)^{2}-1}+\frac{8 i^{2} \omega_{1}|\gamma|^{2}}{n^{2}-1} \\
c_{1}=-\frac{1}{8}+\frac{2 n-1}{(2 n-1)^{2}-1}+\frac{2(n-2)}{(n-2)^{2}-1}, \\
c_{2}=-\frac{1}{2}+(n-2) /\left((n-2)^{2}-1\right) .
\end{gathered}
$$


In deriving (34), we made use of the following relation, which can be derived from (17):

$$
\alpha_{0}^{\prime \prime}=\alpha_{0}\left[\left\{i \omega_{1}+\frac{1}{2}\left(\left|\alpha_{0}\right|^{2}+2|\gamma|^{2}-1\right)\right\}^{2}+\frac{1}{2}\left|\alpha_{0}\right|^{2}\left(\left|\alpha_{0}\right|^{2}+2|\gamma|^{2}-1\right)\right] .
$$

If we multiply both sides of (34) by $\bar{\alpha}_{0}$ and use (17) to derive $\bar{\alpha}_{0} \alpha_{1}^{\prime}=(d / d \tau)\left(\bar{\alpha}_{0} \alpha_{1}\right)+$ $\left(\bar{\alpha}_{0} \alpha_{1}\right)\left[-i \omega_{1}+\frac{1}{2}\left(\left|\alpha_{0}\right|^{2}+2|\gamma|^{2}-1\right)\right]$, then (34) can be written as

$$
\begin{aligned}
\frac{d}{d \tau}\left(\bar{\alpha}_{0} \alpha_{1}\right)+\left(\bar{\alpha}_{0} \alpha_{1}\right)\left[\frac{3}{2}\left|\alpha_{0}\right|^{2}+2|\gamma|^{2}-1\right]+ & \frac{1}{2}\left|\alpha_{0}\right|^{2}\left(\alpha_{0} \bar{\alpha}_{1}\right) \\
& =\frac{\left|\alpha_{0}\right|^{2}}{2 i_{0}} G+\frac{\gamma^{2} \bar{\alpha}_{0}^{4} \delta_{n 2}}{2 \dot{\omega}_{0}} c_{1}+\frac{\gamma \bar{\alpha}_{0}^{5} \delta_{n 5} c_{2}}{2 \dot{\omega}_{0}} .
\end{aligned}
$$

Let us define $w_{1}$ and $w_{2}$ by

$$
\bar{\alpha}_{0} \alpha_{1}=w_{1}+i w_{2} .
$$

Then, using (37), we obtain

$$
\begin{aligned}
& w_{1}^{\prime}+w_{1}\left[2\left|\alpha_{0}\right|^{2}+2|\gamma|^{2}-1\right]=\frac{\left|\alpha_{0}\right|^{2}}{2 \omega_{0}} \operatorname{Im} G+\operatorname{Re} \frac{1}{2 i \omega_{0}}\left[\begin{array}{cc}
\gamma^{2} \bar{\alpha}_{0}^{4} & \delta_{n 2} c_{1} \\
+\gamma \bar{\alpha}_{0}^{5} & \delta_{n 5} c_{2}
\end{array}\right], \\
& w_{2}^{\prime}+w_{2}\left[\left|\alpha_{0}\right|^{2}+2|\gamma|^{2}-1\right]=-\frac{\left|\alpha_{0}\right|^{2}}{2 \omega_{0}} \operatorname{Re} G+\operatorname{Im} \frac{1}{2 i \omega_{0}}\left[\begin{array}{cc}
\gamma^{2} \bar{\alpha}_{0}^{4} & \delta_{n 2} c_{1} \\
+\gamma \bar{\alpha}_{0}^{5} & \delta_{n s} c_{2}
\end{array}\right] \text {. }
\end{aligned}
$$

Now from (24), (25) and (26), it follows that

$$
\begin{aligned}
\lim _{\gamma \rightarrow \infty}\left(2\left|\alpha_{0}\right|^{2}+2|\gamma|^{2}-1\right) & =1-2|\gamma|^{2}, & & |\gamma|^{2}<\frac{1}{2}, \\
& =2|\gamma|^{2}-1, & & |\gamma|^{2}>\frac{1}{2},
\end{aligned}
$$

and

$$
\begin{aligned}
\lim _{r \rightarrow \infty}\left(\left|\alpha_{0}\right|^{2}+2|\gamma|^{2}-1\right) & =0, & & |\gamma|^{2}<\frac{1}{2}, \\
& =2|\gamma|^{2}-1, & & |\gamma|^{2}>\frac{1}{2} .
\end{aligned}
$$

If we take $|\gamma|^{2}<\frac{1}{2}$, which is the case when modulated subharmonics occur, it follows from (39) and (40) that a necessary condition for $\alpha_{1}(\tau)$ to be bounded in $\tau$ is that the term on the right-hand side of (40), which is asymptotically $(\tau \rightarrow \infty)$ constant, must be zero. This condition determines $\omega_{2}$ as

$$
\begin{aligned}
\omega_{2} & =-\frac{1}{16 \omega_{0}}+\frac{|\gamma|^{2}}{2 \omega_{0}}\left[\frac{1}{2}-\frac{2(n+2)}{(n+2)^{2}-1}+\frac{2(n-2)}{(n-2)^{2}-1}\right] \\
& +\frac{|\gamma|^{4}}{2 \omega_{0}}\left[-\frac{1}{2}-\frac{2 n+1}{(2 n+1)^{2}-1}+\frac{2 n-1}{(2 n-1)^{2}-1}+\frac{4(n+2)}{(n+2)^{2}-1}-\frac{4(n-2)}{(n-2)^{2}-1}\right] \\
& +S\left(\omega_{1}\right)
\end{aligned}
$$

where

$$
\begin{aligned}
& S\left(\omega_{1}\right)=0, \quad \omega_{1} \neq 0 \\
& =\frac{1}{16 \omega_{0}} \operatorname{Re}\left[2 \gamma^{2}\left(1-2|\gamma|^{2}\right)^{2} \bar{k}^{4} \delta_{m 2}+\gamma\left(1-2|\gamma|^{2}\right)^{5 / 2} \bar{k}^{3} \delta_{m s}\right], \quad \omega_{1}=0 .
\end{aligned}
$$

It is interesting to note that $\omega_{2}$ is independent of $\omega_{1}$ so that the entrainment for $\omega_{1} \neq 0$ is valid to $O\left(\epsilon^{3}\right)$ at least. In a similar manner to that used in determining $\omega_{2}$, we can 
determine the values $\omega_{k}(k \geq 3)$ uniquely. Finally, we observe that when $\gamma=0$ we obtain the $O\left(\epsilon^{2}\right)$ term in the steady-state frequency for the self-sustained free oscillation of the van der Pol oscillator.

\section{RETERENCES}

[1] T. von Karman, The engineer grapples with nonlinear problems, Bull. Amer. Math. Soc. 46, 615-683, (1940)

[2] S. Lefshetz, Linear and nonlinear oscillations, in Modern mathematics for the engineer, New York, McGraw-Hill, 1956, pp. 7-30

[3] J. J. Stoker, Nonlinear vibrations, Interscience, 1950

[4] N. Minorsky, Nonlinear oscillations, Van Nostrand, 1962

[5] A. Andronov, A. Vitt, and S. Khaikin, Theory of oscillators, Addison-Wesley, 1966

[6] N. Krylov and N. Bogoliuboff, Introduction to nonlinear mechanics, Princeton University Press, 1947

[7] H. Cohen, On subharmonic synchronization of nearly-linear systems, Quart. Appl. Math. 13, 102-105 (1955)

[8] H. Cohen, Subharmonic synchronization for the forced van der Pol equation, in Actes du colloque international des vibrations non-lineaires, Ile de Porquerolles, 1951, P.S.T. 281, French Ministry of Air, 1953, pp. 169-188

[9] J. D. Cole, Perturbation methods in applied mathematics, Blaisdell, 1968 